\title{
Mathematical analysis of a kinetic model for enzymatic cellulose hydrolysis
}

\author{
N. Mohd Jamil \\ Faculty of Industrial Sciences \& Technology, \\ Universiti Malaysia Pahang, Malaysia
}

\begin{abstract}
Biofuel production such as ethanol from lignocellulosic biomass consists of three fundamental processes: pretreatment, enzymatic hydrolysis, and fermentation. Enzymatic hydrolysis uses two types of enzymes simultaneously: endoglucanase I $\left(E_{1}\right)$ and cellobiohydrolase I $\left(\mathrm{CBH}_{1}\right)$, to break the cellulose chains into sugar in the form of cellobiose or glucose. We studied a currently proposed kinetic model for enzymatic hydrolysis of cellulose that uses the population balance equation. The model describes the changes in the cellulose chain length distribution. The complexity of the model makes finding the analytical solution difficult. Therefore, we split the full model into two cases of individual enzyme hydrolysis action and perform mathematical analysis of a single pure enzyme of both cases. The approximate solutions for both cases were derived by employing the asymptotic analysis method. The integrodifferential equation in the first case is solved using Laplace transform. Some significant characteristics are captured. The higher the rate of exposure of cellulose substrates to enzymes, the higher the number of cellulose chains generated from the breakage process. And also, the rate coefficient for $\mathrm{CBH}_{1}$ to locate and thread a reducing end of a cellulose chain is a key factor in bioconversion.
\end{abstract}

Keywords: cellulose, enzymatic hydrolysis, kinetics model, asymptotic analysis, population balance equations, Laplace transform.

\section{Introduction}

Kinetic theory is a way to describe the time evolution of probability distributions of various elementary objects in a system. The scheme is formulated as partial differential equations called kinetic equations for the probability distributions. 
Kinetic equations have been used in describing polymeric fluid flows [1,2], active biological systems [3] and solid materials. Meanwhile, the kinetics equations of enzymatic cellulose hydrolysis in creating ethanol in green energy has been extensively studied, and many kinetic models have been proposed. Some of the models have been derived based on empirical descriptions [4], Michaelis-Menten type equations [5] and experimental observations [6]. Most of these works are successful, at least partially, in tracing the complicated cellulose-cellulase system quantitatively. However, most of the kinetic models do not take into account all important feature e.g. the different action of enzymes, the event of insoluble and soluble cellulose chains, the distribution of cellulose chain lengths, and the time evolution of the biomass particles accessibility to cellulases.

Griggs et al. [7] tackled the major factors mentioned in the previous paragraph by using population balance equations (PBE), a technique that describes the changes in cellulose chain length distribution. Their kinetic model attained computationally efficient result since it not require to working out equations for all chemical species exist in the reacting mixture. For these reasons, the current kinetic model for enzymatic hydrolysis of cellulose proposed by Griggs et al. [7] will be discussed in this paper. The kinetic model depends on the nature of the cellulolytic enzymes employed in the hydrolysis that are Trichoderma reesei endoglucanase I $\left(E_{1}\right)$ and an exoglucanase cellobiohydrolase I $\left(\mathrm{CBH}_{1}\right)$, as well as the structure of cellulose particles. The purposes of both enzymes are to break long cellulose chains into short ones. This feature is also known as depolymerization processes. An understanding of this model is important in designing reaction vessels and optimizing process parameters. This kinetic model provides a deeper understanding, improve predictive capabilities, and ultimately provide more directed and rational approaches for process design and optimization.

\section{Kinetic model formulation}

Cellulose is an insoluble polymer, composed of repeating units of glucose linked by $\beta$ - $(1,4)$-glycosidic bonds and having varied degrees of polymerization [8]. Naturally-occurring cellulosic particles have a wide distribution of chain lengths. The availability of accessibility of the cellulose to enzyme hydrolysis can depend on the chain length [9]. The measure of enzyme-accessible cellulose deviate from the total cellulose in a reaction mixture because of the arrangement of cellulose chains in the cellulose particles.

We treat the cellulosic substrates as "populations" of various chain lengths. We let $P(x)$ be an insoluble cellulose chain comprised of $x$ anhydroglucose units. Here, $x$ is treated as a continuous variable for the sake of simplicity. Suppose $p(x, t)$ is the population distribution of enzyme-accessible cellulose chains (concentration of the unthreaded surface exposed cellulose) of length $x$ at time $t$ in a unit volume. Then, $p(x, t) d x$ is the number of cellulose chains in the length range $(x, x+d x)$ per unit volume at time $t$. 
With the distribution function $p(x, t)$, one can define the $n$th moment of the distribution function as $p^{(n)}(t)=\int_{0}^{\infty} x^{n} p(x, t) d x$. The zeroth moment, $p^{(0)}$, gives the total number of cellulose chains at time $t$ in a unit volume. The first moment $p^{(1)}$ is the total number of monomeric glucans comprising the cellulose chains at time $t$. The number-averaged chain length is denoted by $x_{N}=p^{(1)} / p^{(0)}$. The massaveraged chain length is the ratio of the second to first moments $x_{M}=p^{(2)} / p^{(1)}$, where the number density is proportional to the mass of the cellulose chain by assuming the monomeric glucan unit has a constant mass [7].

The set of modelling equations as proposed in [7] are as follows:

$$
\begin{aligned}
\frac{d p(x, t)}{d t}= & -k_{f}^{C B H} E_{s}^{C B H} p(x, t)+2 \int_{x}^{\infty} k_{h}^{E G}(y) E_{s}^{E G} p(y, t) \Omega(x, y) d y \\
& -k_{h}^{E G}(x) E_{s}^{E G} p(x, t)+\int_{x}^{\infty} k_{h}^{E G}(y) E_{s}^{E G} p_{B}(y, t) \Omega(x, y) d y \\
& -\left(1-\frac{R_{0}}{R(t)}\right) \frac{p_{i}(x)}{p_{i}^{(1)}} r_{l o s s}^{(1)}, \\
\frac{d p_{B}(x, t)}{d t}= & k_{f}^{C B H} E_{s}^{C B H} p(x, t)+k_{h}^{C B H}\left[p_{B}\left(x+x_{2}\right)-p_{B}(x, t)\right] \\
& +\int_{x}^{\infty} k_{h}^{E G}(y) E_{s}^{E G} p_{B}(y, t) \Omega(x, y) d y-k_{h}^{E G}(x) E_{s}^{E G} p_{B}(x, t), \\
\frac{d R}{d t}= & \frac{r_{l o s s}^{(1)}}{2 n \pi \rho R L}, R \geq R_{0} .
\end{aligned}
$$

where $p_{B}(x, t)$ is the distribution of $\mathrm{CBH}_{1}$-threaded cellulose chain with length $x$, and $p_{i}(x)$ is the population distribution of inaccessible cellulose. $k_{f}^{C B H}$ is the rate for $\mathrm{CBH}_{1}$ to adsorb on the cellulose chains, $k_{h}^{C B H}$ is the rate for $\mathrm{CBH}_{1}$ finds and threads the $\mathrm{CBH}$-threaded cellulose chains which also known as the rate of processive hydrolysis, $k_{h}^{E G}(x)$ is the rate for $\mathrm{EG}_{1}$ finds and cleave the cellulose chains of length $x, E_{s}^{E G}$ and $E_{s}^{C B H}$ are surface-adsorbed $\mathrm{EG}_{1}$ and $\mathrm{CBH}_{1}$, respectively. The term $\Omega(x, y)$ is known as the breakage kernel. We assume that the pretreated biomass particles are monodisperse cylindrical shaped with radius $R$ and length $L$. Denote $R_{0}$ as the thickness of single cellulose chains and $r_{\text {loss }}$ is the rate of loss of cellulose during enzymatic hydrolysis.

Due to the complexity of the system, which arises from the integrodifferential equations makes finding the analytical solution difficult. To solve the system of equations, we perhaps have to resort to numerical methods that can incur 
significant computational cost. Our strategy here is to look for reduced order models by ignoring significantly small terms in the governing system of equations. We employ the asymptotic analysis for dynamical systems to reduce the complex model to a set of simple equations. The resulting approximate solutions of a simple model may not fully capture all the details of the complex system. However, it usually captures some important characteristics and provide insights into potential dynamical and chemical mechanisms and their dependence on parameters. This paper will present the mathematical analysis of the independent action of $\mathrm{EG}_{1}$ or $\mathrm{CBH}_{1}$ from the kinetic model that will be formulated as Model I and Model II, respectively. The goal is to ensure that mathematical results are consistent with physical requirements.

Given $\Omega(x, y)=1 / y$ and the rate coefficient $k_{h}^{E G}(x)=0.002 \tilde{k}_{h}^{E G} x$ which dictate more frequent action by $\mathrm{EG}_{1}$ on longer chains. We introduce the dimensionless variables: $\tau=\frac{t}{t_{0}}, \widehat{p}(x, \tau)=\frac{p(x, t)}{p^{(0)}(0)}, \widehat{p_{B}}(x, \tau)=\frac{p_{B}(x, t)}{p^{(0)}(0)}$, and $\widehat{R}(\tau)=\frac{R}{R_{0}}$ where $t_{0}$ is the characteristic time and $p^{(0)}(0)$ is the initial total population density. $x$ has already been made dimensionless so that it represents number of andhydroglucose units. Suppose $p(x, 0)=p^{(0)}(0) g(x)$ where $g(x)$ is the initial size distribution. Hence, $\hat{p}(x, \tau=0)=g(x)$ and $\widehat{R}(\tau=0)=R_{i n} / R$. We take the thickness of a single glucan $R_{0}=1 \mathrm{~nm}$ and $R_{i n}=100 \mathrm{~nm}$. Therefore, $\widehat{R}(\tau=0)=100$.

The full model is split into two limiting cases of individual enzyme hydrolysis i.e. pure action of $\mathrm{EG}_{1}$ and pure action of $\mathrm{CBH}_{1}$ so called Model I and Model II, respectively. Model I describes the independent action of $\mathrm{EG}_{1}$ where we assume that the coefficients in the full model related to $\mathrm{CBH}_{1}$ are very small as follows:

$$
\begin{aligned}
\frac{d \hat{p}(x, \tau)}{d \tau}= & 0.004 \tilde{k}_{h}^{E G} E_{s}^{E G} t_{0} \int_{x}^{\infty} \hat{p}(y, \tau) d y-0.002 \tilde{k}_{h}^{E G} E_{s}^{E G} t_{0} x \hat{p}(x, \tau) \\
& -\left(1-\frac{1}{\hat{R}}\right) \frac{p_{i}(x)}{p_{i}^{(1)}} r_{\text {loss }}^{(1)} \frac{t_{0}}{p^{(0)}(0)}, \\
\frac{d \hat{R}}{d \tau} & =\frac{r_{\text {loss }}^{(1)} t_{0}}{2 n \pi \rho R R_{0}^{2} L}, \widehat{R} \geq 1 .
\end{aligned}
$$

Likewise, Model II deal with individual action of $\mathrm{CBH}_{1}$ enzyme by assuming that the coefficients associated with $\mathrm{EG}_{1}$ are very small as follows: 


$$
\begin{aligned}
\frac{d \hat{p}(x, \tau)}{d \tau} & =-k_{f}^{C B H} E_{s}^{C B H} t_{0} \hat{p}(x, \tau)-\left(1-\frac{1}{\hat{R}}\right) \frac{p_{i}(x)}{p_{i}^{(1)}} r_{\text {loss }}^{(1)} \frac{t_{0}}{p^{(0)}(0)}, \\
\frac{d \widehat{p_{B}}(x, \tau)}{d \tau} & =k_{f}^{C B H} E_{s}^{C B H} t_{0} \hat{p}(x, \tau)+k_{h}^{C B H} t_{0}\left[x_{2} \frac{\partial \widehat{p_{B}}}{\partial x}+\frac{x_{2}^{2}}{2} \frac{\partial^{2} \widehat{p_{B}}}{\partial x^{2}}\right], \\
\frac{d \hat{R}}{d \tau} & =\frac{r_{\text {loss }}^{(1)} t_{0}}{2 n \pi \rho R R_{0}^{2} L}, \widehat{R} \geq 1 .
\end{aligned}
$$

The mathematical analysis of Model I and Model II will be conducted separately in the next section.

\section{Model I (independent action of EG1)}

In this section, we will focus to study how random-chain scission by $\mathrm{EG}_{1}$ changes a population of cellulose chains. Let $t_{0}=\frac{1}{\tilde{k}_{h}^{E G} p^{(0)}(0)}$ and we introduce the dimensionless parameter groups as following: $\tilde{a}=\frac{E_{s}^{E G}}{p^{(0)}(0)}, \quad \tilde{b}=\frac{p_{i}}{p_{i}^{(1)}}$, $\tilde{c}=-\frac{r_{\text {loss }}^{(1)}}{\tilde{k}_{h}^{E G}\left(p^{(0)}(0)\right)^{2}}$, and $\tilde{d}=\frac{p^{(0)}(0)}{2 n \pi \rho R_{0}^{2} L}$. The reduction in the number of parameters makes theoretical manipulations easier, for the equations are less cluttered. Take $\tilde{a}=500$. We can dictate that $p_{i}^{(1)} \gg p_{i}(x)$ since $p_{i}^{(1)}$ always give us a large number of glucan units of inaccessible cellulose chains (located in the interior of a cylinder) compared to the number of inaccessible cellulose chains of length $x$. We also argue that the rate of loss of cellulose of length $x$ due to enzymatic hydrolysis, $r_{\text {loss }}^{(1)}$, is small compared to $p^{(0)}(0)$ because the initial total number of cellulose chains give a very large value. Hence, we denote the small terms $\tilde{b}$ and $\tilde{c}$ as $\varepsilon$ and omitting the 'hat' for notational simplicity therefore we obtained:

$$
\begin{aligned}
\frac{d p(x, t)}{d t} & =2 \int_{x}^{\infty} p(y, t) d y-x p(x, t)-\left(1-\frac{1}{R}\right) \varepsilon^{2}, \\
\frac{d R}{d t} & =-\frac{\tilde{d} \varepsilon}{R}, R \geq 1
\end{aligned}
$$

subject to initial conditions $p(x, 0)=g(x)$ and $R(t=0)=100$. From this system, we can solve $R$ independently, yields $R=\sqrt{10000-2 \tilde{d} \varepsilon t}$. This equation 
describes the behavior of the radius of the cellulose particle. It is obvious that this equation agrees to our expectation that the radius of particles will be decreasing over time during the hydrolysis. These phenomena explain the effect of the enzyme which breaking the polymer chains on the surface of cylinder particles and make the interior part exposed. Further, the enzyme will react on the exposed part. This process occurs repeatedly until the cylinder particles become thin. In order to get insight of how fast the radius will decrease, we fixed $\varepsilon=0.001$ and varied the value of $\tilde{d}$ by setting $\tilde{d}=1000,2000,3000$ as illustrated in Figure 1. It showed an increased in the rate of radius thinning with increased $\tilde{d}$.

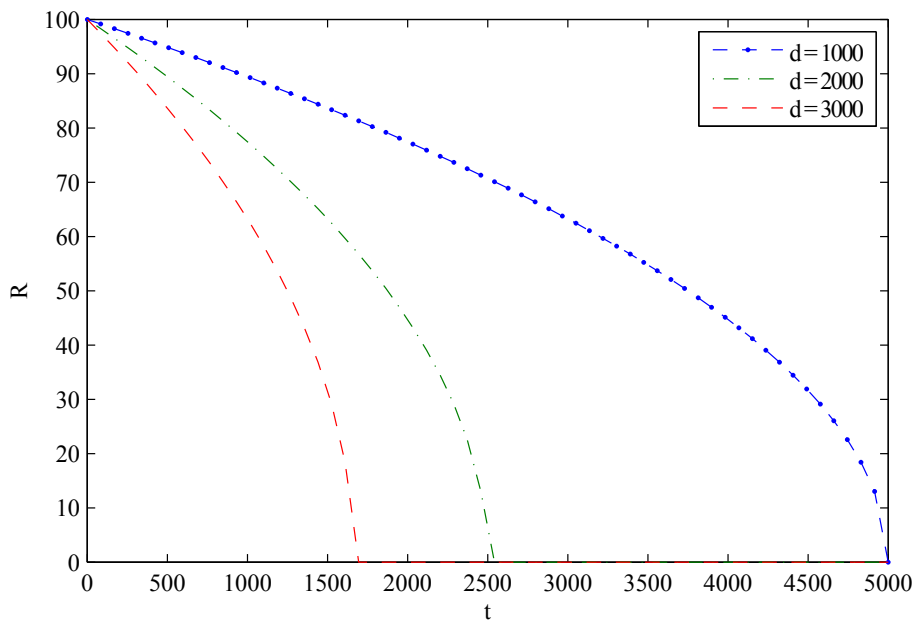

Figure 1: The evolution of radius of cellulose particles during enzymatic hydrolysis. The radius thinning increased with increased $\tilde{d}$.

Next, by denoting $\zeta(t, \tilde{d}):=1-\frac{1}{R(t)}$, eqn (1) can be rewritten as

$$
\frac{d p(x, t)}{d t}=2 \int_{x}^{\infty} p(y, t) d y-x p(x, t)-\zeta(t, \tilde{d}) \varepsilon^{2} .
$$

Note that $0 \leq \zeta(t, \tilde{d})<1$ since $R \geq 1$. In general, analytical solutions to population balance equations are very difficult to obtain. The approach to solving integrodifferential equations is usually to apply the Laplace transform method with hopes that an inverse to the transfer function is readily available.

The integrodifferential equation in eqn (2) can be transformed in a partial differential equation using Laplace transformation in the particle size variable. We define the Laplace transform of the particle size distribution with respect to the 
length variable as $\quad \psi(s, t)=\mathcal{L}\{p(x, t)\}=\int_{0}^{\infty} p(x, t) e^{-s x} d x$. Since $\psi(0, t)=\int_{0}^{\infty} p(x, t) d x$ equal to the zeroth moment of $p(x, t)$, we set $\psi(0, t)=e^{-t}+t+1$ based on a formulation in [10]. Hence, by taking the Laplace transform of eqn (2), we obtain

$$
\frac{\partial \psi(s, t)}{\partial t}=\frac{2}{s}\left(1+t+e^{-(t+s)}-\psi\right)+\frac{\partial \psi}{\partial s}+\frac{\zeta(t, \tilde{d}) \varepsilon^{2}}{s},
$$

with boundary conditions $\psi(0, t)=e^{-t}+t+1$ and $\psi(\infty, t)=0$. Taking Laplace transform of the initial condition of $p(x, t)$, we have the initial condition for eqn (3) given by

$$
\psi(s, 0)=\int_{0}^{\infty} p(x, 0) e^{-s x} d x=\int_{0}^{\infty} g(x) e^{-s x} d x=\mathcal{L}\{g(x)\} .
$$

If the initial particle length distribution is monodisperse with dimensionless size unity i.e. $g(x)=\delta(x-1)$, then $\psi(s, 0)=e^{-s}$. Since there is a parameter in the equation that is relatively small, we are interested in using what is known as asymptotic expansions to find approximate solutions of the differential equation. We used asymptotic method to provide a reasonably accurate expressions for the solution. In other words, for small $\varepsilon$, it is assumed that $\psi \sim \psi_{0}(s, t)+\varepsilon \psi_{1}(s, t)+\varepsilon^{2} \psi_{2}(s, t)+\ldots$ The solution for $\mathrm{O}(1)$ is $\psi_{0}(s, t)=\frac{\left(t^{2}+2 t\right)}{s+t}-\frac{t^{2}}{(s+t)^{2}}+e^{-(s+t)}$. We recognize this solution satisfy all boundary conditions. The next term is important as it gives a measure of the error. For $\mathrm{O}(\varepsilon)$ and $\mathrm{O}\left(\varepsilon^{2}\right), \psi_{1}(s, t)=0$ and $\psi_{2}(s, t)=\frac{\zeta}{2}\left(1-\frac{s^{2}}{(s+t)^{2}}\right)$, respectively. Therefore we have that

$$
\psi(s, t) \sim \frac{\left(t^{2}+2 t\right)}{s+t}-\frac{t^{2}}{(s+t)^{2}}+e^{-(s+t)}+\varepsilon^{2} \frac{\zeta}{2}\left(1-\frac{s^{2}}{(s+t)^{2}}\right)
$$

Then, the Laplace transform (eqn (4)) can be inverted to give the solution to the original problem by taking $\varepsilon \rightarrow 0$ :

$$
p(x, t)=e^{-t} \delta(x-1)+\left[2 t+t^{2}(1-x)\right] e^{-x t} .
$$


That eqn (5) is a solution to eqn (2) can be verified by substitution. The moments follow by integrating eqn (5) yield $p^{(0)}(t)=e^{-t}+t+1$ and $p^{(1)}(t)=e^{-t}+1$. The evolution of the zeroth and first moments are illustrated in Figure 2. Results clearly show that the zeroth moment of the particle size distribution is increasing, since, during breakage, the total number of particles is increasing. The behavior of the first moment indicates that the total number of glucan units is conserved until end of the hydrolysis process.

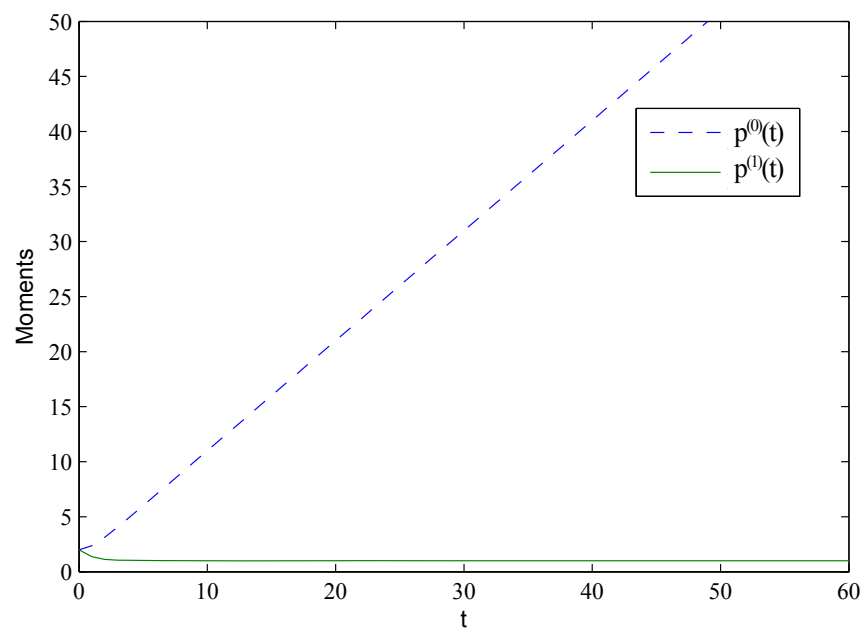

Figure 2: Time evolution of the zeroth and the first moments predicted by the approximate analytical solution. The number of particles increase while the number of glucan units is conserved.

\section{Model II (independent action of $\mathrm{CBH}_{1}$ )}

The case of random scission by $\mathrm{EG}_{1}$ has been analyzed extensively from the mathematical point of view in the previous section. In this section, we account for two glucans depolymerization in the enzymatic hydrolysis of cellulose by $\mathrm{CBH}_{1}$, an enzyme that performs processive chain-end scission. Here we put $t_{0}=\frac{1}{k_{f}^{C B H} p^{(0)}(0)}$ and the dimensionless group of parameters as follows:

$$
\tilde{b}=\frac{p_{i}}{p_{i}^{(1)}}, \tilde{d}=\frac{p^{(0)}(0)}{2 n \pi \rho R_{0}^{2} L}, \tilde{e}=\frac{E_{s}^{C B H}}{p^{(0)}(0)}, \tilde{f}=-\frac{r_{\text {loss }}^{(1)}}{k_{f}^{C B H}\left(p^{(0)}(0)\right)^{2}}, \tilde{g}=\frac{k_{h}^{C B H}}{k_{f}^{C B H} p^{(0)}(0)}
$$

We omitted the 'hat' for notational simplicity, therefore we obtained 


$$
\begin{aligned}
\frac{d p(x, t)}{d t} & =-\tilde{e} p(x, t)+\left(1-\frac{1}{R}\right) \tilde{b} \tilde{f}, \\
\frac{d p_{B}(x, t)}{d t} & =\tilde{e} p(x, t)+\tilde{g}\left[x_{2} \frac{\partial p_{B}}{\partial x}+\frac{x_{2}^{2}}{2} \frac{\partial^{2} p_{B}}{\partial x^{2}}\right], \\
\frac{d R}{d t} & =-\frac{\tilde{f} \tilde{d}}{R}, R \geq 1 .
\end{aligned}
$$

We set $\tilde{e}=1$ and $\tilde{g}=1$ as a reference case and the variation of these values can be found in Parameter Analysis subsection. With the same reason as in Model I, we assume $\tilde{b}$ and $\tilde{f}$ are small terms so that we can represent them as $\varepsilon$. Hence, by putting the above setting,

$$
\begin{aligned}
\frac{d p(x, t)}{d t} & =-p(x, t)+\left(1-\frac{1}{R}\right) \varepsilon^{2}, \\
\frac{d p_{B}(x, t)}{d t} & =p(x, t)+x_{2} \frac{\partial p_{B}}{\partial x}+\frac{x_{2}^{2}}{2} \frac{\partial^{2} p_{B}}{\partial x^{2}}, \\
\frac{d R}{d t} & =-\frac{\varepsilon \tilde{d}}{R}, R \geq 1 .
\end{aligned}
$$

with the initial condition $p(x, 0)=g(x)$ and $R(t=0)=100$. We solved $R$ independently, and we attained the same result for particle radius as discussed for Model I. We conclude that $\mathrm{EG}_{1}$ and $\mathrm{CBH}_{1}$ enzymes are both responsible for the reduction in radius of cellulose particles independently.

Next, we solve for $p(x, t)$. By denoting $\zeta(t, \tilde{d}):=1-\frac{1}{R(t)}$, we may write $\frac{\partial p(x, t)}{\partial t}=-p(x, t)+\zeta(t, \tilde{d}) \varepsilon^{2} . \quad$ Let $\quad p(x, 0)=g(x)=\delta(x-1) . \quad$ Laplace transforms are not suited to apply here since integrodifferential term does not appear in this model. By using asymptotic approximation, we assume $p \sim p_{0}(x, t)+\varepsilon p_{1}(x, t)+\varepsilon^{2} p_{2}(x, t)+\ldots \quad$ For $\quad \mathrm{O}(1), \quad p_{0}(x, t)=\delta(x-1) e^{-t}$ whereas for $\mathrm{O}(\varepsilon), \quad p_{1}(x, t)=0$. For $\mathrm{O}\left(\varepsilon^{2}\right), \quad p_{2}(x, t)=\zeta\left(1-e^{-t}\right)$ and $p_{n}(x, t)=0$ for $n \geq 3$. Therefore we have $p(x, t) \sim \delta(x-1) e^{-t}+\varepsilon^{2} \zeta\left(1-e^{-t}\right)$. By taking $\varepsilon \rightarrow 0$,

$$
\frac{\partial p_{B}(x, t)}{\partial \tau}=x_{2} \frac{\partial p_{B}}{\partial x}+\frac{x_{2}^{2}}{2} \frac{\partial^{2} p_{B}}{\partial x^{2}}+\delta(x-1) e^{-t} .
$$

The boundary conditions $\lim _{x \rightarrow \pm \infty} \frac{\partial p_{B}}{\partial x}=0$ were used. Initial particle size distribution and their evolution can be represented by a gamma distribution 
$p_{B}(x, 0)=\frac{p_{B}^{(0)}}{\beta^{\alpha} \Gamma(\alpha)} x^{n-1} e^{-x / \beta}$, [7] where $\alpha, \beta$ and $p_{B}^{(0)}$ determine the mean, width, and total size of the distribution. In this paper, the parameters $\alpha=101$, $\beta=1.5$ and $p_{B}^{(0)}=900$ was used for an example simulation. We obtained the result for Model II using pdepe solver in MATLAB. The progression of chain-end scission is shown in Figure 3. The maximum peak moves to the left as time passed by which indicates the cellulose chains has been reduced to shorter chains.

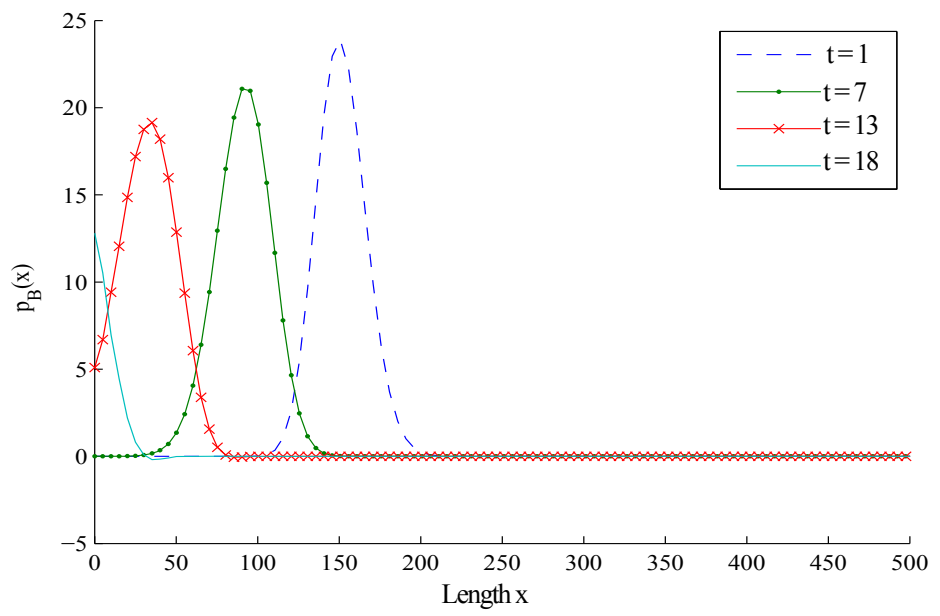

Figure 3: The evolution of $p_{B}$ distribution undergoing chain-end scission with $\tilde{e}=1$ and $\tilde{g}=1$.

\subsection{Parameter analysis}

We performed parametric sensitivity analysis to study the influence of $k_{f}^{C B H}$ (rate coefficient for $\mathrm{CBH}_{1}$ to locate and thread a reducing end of a cellulose chain) and $k_{h}^{C B H}$ (rate coefficient for processive hydrolysis by $\mathrm{CBH}_{1}$ ) by varying the value of $\tilde{e}$ and $\tilde{g}$ in eqn (6), respectively. We study the sensitivity of parameter $\tilde{g}$ in Model II based on different setting: (a) $\tilde{g}=0.5$ and (b) $\tilde{g}=2$. The variation of this parameter was used to determine the change in $k_{h}^{C B H}$. It should be noted that for these two cases, parameter $\tilde{e}$ is fixed to 1 . The resulting effect of the $k_{h}^{C B H}$ on the particle size distribution of $p_{B}$ can be seen in Figure 4 and 5. It becomes obvious that the performance of the distribution improves with higher $k_{h}^{C B H}$ loading. Next, we fixed the value of $\tilde{g}$ to 1 and varied $\tilde{e}$. The result shows that the conversion of insoluble cellulose chains to soluble sugars is not sensitive to the $k_{f}^{C B H}$ values. 


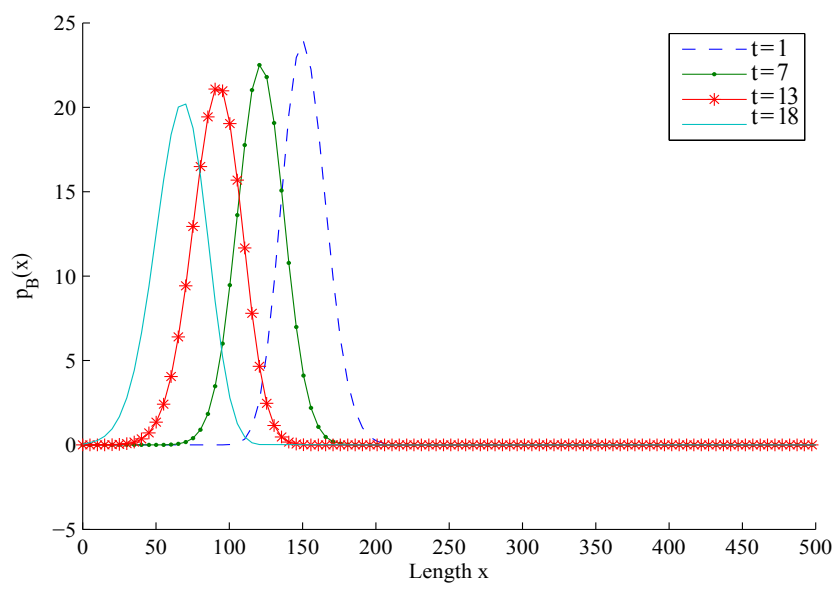

Figure 4: The evolution of $p_{B}$ distribution undergoing chain-end scission with $\tilde{e}=1$ and $\tilde{g}=0.5$.

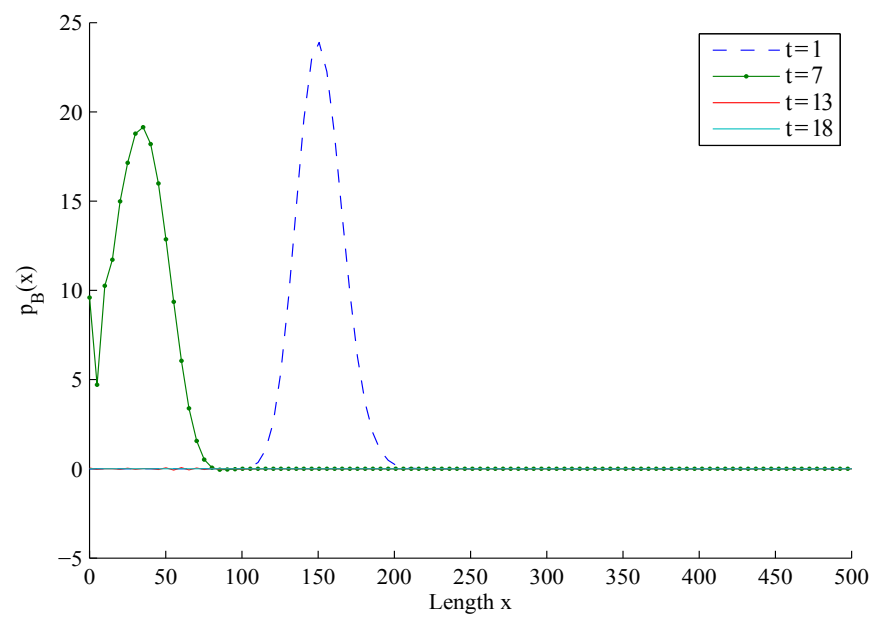

Figure 5: Time evolution of $p_{B}$ distribution undergoing chain-end scission with $\tilde{e}=1$ and $\tilde{g}=2$.

\section{Conclusion}

We analyzed two separate models for enzymatic hydrolysis of cellulose: Model I is for independent action of $\mathrm{EG}_{1}$ and Model II is for pure $\mathrm{CBH}_{1}$ action. By using asymptotic analysis, we derived the approximation solutions for both models. As a result, both models gave the same behavior where the number of cellulose chains 
increases over time. This is due to the effect of both enzymes in breaking the cellulose chains into smaller ones so that more cellulose particles are generated. The higher the rate of exposure of cellulose substrates to enzymes, the higher the number of cellulose chains generated from the breakage process. And also, the rate coefficient for $\mathrm{CBH}_{1}$ to locate and thread a reducing end of a cellulose chain is a key factor in bioconversion.

\section{References}

[1] S. F. Edwards and M. Doi, The theory of polymer dynamics, Clarendon, Oxford, 1986.

[2] R. B. Bird, R. C. Armstrong and O. Hassager, Dynamics of polymeric liquids. Volume 1: fluid mechanics, A Wiley-Interscience Publication, John Wiley Sons, 1987.

[3] J.-F. Joanny, K. Kruse, J. Prost and S. Ramaswamy, "The actin cortex as an active wetting layer," The European Physical Journal E, vol. 36, no. 5, pp. 1-6, 2013.

[4] P. Bansal, M. Hall, M. J. Realff, J. H. Lee and A. S. Bommarius, "Modeling cellulase kinetics on lignocellulosic substrates," Biotechnology advances, vol. 27, no. 6, pp. 833-848, 2009.

[5] M. Okazaki and M. Moo-Young, "Kinetics of enzymatic hydrolysis of cellulose: analytical description of a mechanistic model," Biotechnology and bioengineering, vol. 20, no. 5, pp. 637-663, 1978.

[6] L. Fan and Y.-h. Lee, "Kinetic studies of enzymatic hydrolysis of insoluble cellulose: derivation of a mechanistic kinetic model," Biotechnology and bioengineering, vol. 25, no. 11, pp. 2707-2733, 1983.

[7] A. J. Griggs, J. J. Stickel and J. J. Lischeske, "A mechanistic model for enzymatic saccharification of cellulose using continuous distribution kinetics I: depolymerization by EGI and CBHI," Biotechnology and Bioengineering, vol. 109, no. 3, pp. 665-675, 2012.

[8] K. Igarashi, A. Koivula, M. Wada, S. Kimura, M. Penttil and M. Samejima, "High speed atomic force microscopy visualizes processive movement of Trichoderma reesei cellobiohydrolase I on crystalline cellulose," Journal of Biological Chemistry, vol. 284, no. 52, pp. 36186-36190, 2009.

[9] K. M. Kleman-Leyer, M. SiiKa-Aho, T. T. Teeri and T. K. Kirk, "The Cellulases Endoglucanase I and Cellobiohydrolase II of Trichoderma reesei Act Synergistically To Solubilize Native Cotton Cellulose but Not To Decrease Its Molecular Size.," Applied and environmental microbiology, vol. 62, no. 8, pp. 2883-2887, 1996.

[10] R. M. Ziff and E. McGrady, "The kinetics of cluster fragmentation and depolymerisation," Journal of Physics A: Mathematical and General, vol. 18 , no. 15 , p. $3027,1985$. 